\section{POSTER 037.}

\section{Base-Station Communication: Are Residents as Efficient as Faculty?}

Robert L. Norton, MD, ${ }^{*}$ Brian Hoyt, MSIV

* Department of Emergency Medicine

+ School of Medicine

Oregon Health Sciences University, Portland, Oregon USA

Purpose: Some EMS systems use resident (RES) physicians to provide on-line medical control (OLMC). Resident inexperience may result in less efficient communication.

Objectives: To compare EM faculty physicians (FAC) response to EM senior RES. To determine factors associated with timeefficient OLMC communication. Design: Retrospective cohort for consecutive six months. Setting: A single base-station university hospital staffed by EM FAC (3,300 calls/yr answered by either FAC or RES). Measurements: On-line medical control audio tapes and written records of radio and telephone calls were reviewed. Paramedics (EMT-P) were required to begin OLMC calls with specified information (INFO) (e.g., vital signs, history, treatment). Times were measured using tape deck clock and were reported in mean seconds. CALLTIME = mean time from start to end of physician communication with EMT-P. TOTALTIME = CALLTIME + mean notification time of receiving hospital.

Results: A total of 989 calls were analyzed. There was no significant difference in mean CALLTIME (206 vs 201) or TOTALTIME (246 vs 244) for FAC vs RES $(p=0.71 ; p=0.87)$. Emergency medical services paramedic supervising FAC (228) had significantly shorter TOTALTIME than nonsupervising FAC (254) $(p=0.034)$. Longer TOTALTIME for all physicians was associated with pulse $(\mathrm{P})>100 \mathrm{bpm}$, systolic BP $<90 \mathrm{mmHg}$, altered mental status and number of questions from physician about missing guideline information. The most common missing INFO data not initially provided by the EMT-P were ETA, receiving hospital, respiratory rate (RR), $\mathrm{P}$ and BP. Longer TOTALTIMEs were associated with physicians summarizing information already given or educating the EMT-P on-line, but not for clarifying drug orders or directing nondrug treatment.

Conclusion: Residents are as efficient as FAC in answering OLMC calls. Information not initially given by EMT-P affects the call duration. Improved training of EMT-P for better reporting of INFO and fewer questions from OLMC physicians for INFO already provided may result in more efficient calls.

\section{POSTER 046. \\ Is Contact with On-line Medical Control Necessary for Prehospital Administration of Diazepam?}

Robert L. Norton, MD, * Scott Milne, MD, S. Filarski, MD

Department of Emergency Medicine, Oregon Health Sciences University, Portland, Oregon and Department of Emergency Medicine, University of Cincinnati, Cincinnati, Ohio USA

Background: Base-station physician response is costly, timeconsuming, often not reimbursable, and may not be necessary, even for controlled drugs, if most requests are approved.

Objectives: To determine the frequency of on-line medical control (OLMC) refusal of requests to administer diazepam and to determine whether OLMC physicians are consistent in their response to paramedic requests.

Methods: Design: Retrospective cohort analysis of all EMS online diazepam requests during a 21-month period. Setting: A single base station staffed by EM supervising physicians (SP) (3,300 calls/yr). Participants: Patients for whom diazepam was requested (under any circumstance) by field paramedics.

Results: Requests for diazepam represented $347(6 \%)$ of the 5,790 on-line calls, of which $38(11 \%)$ were refused. There was no significant difference in mean patient age, gender, mean presenting vital signs, or seizure type between refused $(n=38)$ or approved $(n=309)$ diazepam requests. Factors associated more frequently with refusal of diazepam requests compared to approval were an ETA $<4$ minutes $(16 \%$ vs $4 \%, p<0.05)$ and combative patients not seizing at the time of the request $(8 \%$ vs $1 \%, p<0.05$ ). Factors associated more commonly with approval of diazepam were seizure activity at time of request $(66 \%$ vs $24 \%, p<0.001$ ), depressed level of consciousness (29\% vs $16 \%$, $p<0.05)$ and requests between midnight and 0800 (24\% vs $8 \%$, $p<0.05$ ). The odds ratio (OR) for 12 SPs answering $\geq 10$ calls ranged from 0.19 to 3.09 . The OR $95 \%$ confidence interval of only one physician indicated a tendency to refuse diazepam. Of the 10 refusals for that physician, four patients had ETAs $<4$ min., four patients were not seizing at time of contact, and one patient had focal seizures.

Conclusion: On-line medical control physicians were consistent in their responses to requests by paramedics to administer diazepam. Since refusals of paramedic requests were uncommon, standing orders without mandatory call in for use of diazepam should be considered. 